\title{
Aortic Valve Disease, CTCAE
}

National Cancer Institute

\section{Source}

National Cancer Institute. Aortic Valve Disease, CTCAE. NCI Thesaurus. Code C143290.

A disorder characterized by a defect in aortic valve function or structure. 